\title{
Energy Spectra of Rossby and Gravity Waves
}

\author{
Koji Terasaki ${ }^{1}$, H. L. Tanaka ${ }^{1}$, and N. Žagar ${ }^{2}$ \\ ${ }^{1}$ Center for Computational Sciences, University of Tsukuba, Tsukuba, Japan \\ ${ }^{2}$ University of Ljubljana and Center of Excellence Space-SI, Ljubljana, Slovenia
}

\begin{abstract}
The characteristics of energy spectrum based on 3D normal mode energetics are investigated with the global analysis dataset provided by Japan Meteorological Agency (JMA) with the resolution of TL959L60. The energy spectrum of gravity modes exactly follows the $-5 / 3$ power law in the synoptic and mesoscales. In the synoptic scale, the spectral slope of total energy follows the -3 power law because Rossby waves are dominant compared to gravity waves. The energy level of gravity modes becomes larger than that of Rossby modes around the zonal wavenumber $k=80$. This scale corresponds to $350 \mathrm{~km}$ in $45^{\circ}$ circle. The total energy spectrum does not show a clear transition from -3 power slope to $-5 / 3$ power slope because the energy level of Rossby and gravity modes become comparable near the transition wavenumbers.
\end{abstract}

\section{Introduction}

A number of studies related to the spectral energy slope and its mechanism have been conducted by many researchers for the general circulation of the atmosphere. Nastrom and Gage (1985) pointed out from the results of aircraft observations that the energy spectra of zonal wind, meridional wind, and potential temperature obey the $k^{-3}$ power law in the synoptic scale and the $k^{-5 / 3}$ power law in the mesoscale, respectively.

The energy spectrum of the large scale motion is characterized by the $k^{-3}$ law from synoptic to sub-synoptic scales (Wiin-Nielsen 1967; Boer and Shepherd 1983; Nastrom et al. 1984). Using a dimensional analysis, Kraichnan (1967) predicted the $k^{-3}$ power law for $2 \mathrm{D}$, isotropic and homogeneous turbulence in a forward enstrophy cascading inertial subrange on the short-wave side of the scale of energy injection. Tung and Orland (2003) showed that not only enstrophy but also energy cascade down from the synoptic to mesoscales. The downscale energy cascade is responsible for a $k^{-5 / 3}$ spectrum on the short wave side where the energy cascade exceeds the enstrophy cascade. The $k^{-5 / 3}$ power law has previously been predicted theoretically by Kolmogorov (1941) for $3 \mathrm{D}$ homogeneous and isotropic turbulence, producing a downscale energy flux.

Koshyk and Hamilton (2001) examined the differences in the horizontal kinetic energy spectrum using the GFDL (Geophysical Fluid Dynamics Laboratory) SKYHI model with horizontal grid spacing of $35 \mathrm{~km}$. They confirmed that the spectrum follows the $k^{-3}$ power law for wavelengths between about 5000 and $500 \mathrm{~km}$ and $k^{-5 / 3}$ power law at small wavelengths. Hamilton et al. (2008) examined the horizontal spectrum of wind variance in experiments conducted with the Atmospheric GCM for the Earth Simulator (AFES), and found that the control version of AFES run at T639 horizontal spectral resolution, which employs a slightly filtered version of realistic topography, simulates a kinetic energy spectrum that compares well at large scales with global reanalyses and, at smaller scales, with available aircraft observations near the tropopause level. Takahashi et al. (2006) showed with the result from the simulation using AFES that the $k^{-3}$ and $k^{-5 / 3}$ spectra are produced by the atmospheric dynamics. Kitamura and Matsuda (2006) investigated the characteristics of the energy spectra of

Corresponding author: Koji Terasaki, Center for Computational Science, University of Tsukuba, 1-1-1 Tennoudai, Tsukuba 305-8577, Japan. E-mail: koji@ccs.tsukuba.ac.jp. (C2011, the Meteorological Society of Japan. the vortical and gravitational modes using their model which assumes a nonhydrostatic, incompressible Boussinesq fluid on $f$-plain. They found that the downscale energy cascade associated with the divergent modes plays an important role in the formation of the mesoscale $k^{-5 / 3}$ spectrum. Kitamura and Matsuda (2010) conducted a notable numerical experiments in relation to the mesoscale energy cascade process in the free atmosphere. According to their results, the nonlinear interactions between geostrophic and gravitational modes play a crucial role in the downscale energy cascades for forming the $k^{-5 / 3}$ spectrum. Terasaki et al. (2009) showed the kinetic energy spectra for zonal wind and vertical wind components simulated by NICAM (Non-hydrostatic ICosahedral Atmospheric Model) with horizontal grid spacing from $224 \mathrm{~km}$ to $3.5 \mathrm{~km}$. They confirmed that the spectra of zonal wind components follows $k^{-5 / 3}$ law in the mesoscale for $7.0 \mathrm{~km}$ and $3.5 \mathrm{~km}$ experiments. The energy spectrum of the vertical wind in the zonal wavenumber domain was found to be white noise, which all the waves have the same energy level, i.e., which shows an impotant implication of wave saturation, especially for the gravity waves.

Tanaka (1985) and Tanaka and Kung (1988) studied the atmospheric energy spectrum expanding the atmospheric data with 3D normal mode functions. This method can divide the horizontal wind field and geopotential into Rossby and gravity modes by expanding with Hough functions, which can be obtained as a eigen function of linearized horizontal structure equations. Terasaki and Tanaka (2007b) investigated the energetics of atmospheric general circulation using JRA-25 (Japanese 25-year Reanalysis) and ERA-40 (ECMWF 40-year Reanalysis) datasets, and found that the $k^{-3}$ spectrum can be seen in the both datasets, but could not obtain the transition of energy slope from $k^{-3}$ power law to $k^{-5 / 3}$ power law. The energy level in the zonal wavenumber domain drops around the zonal wavenumber $k=20$ due to the low horizontal resolution. Žagar et al. $(2009,2010)$ showed the energy spectra of the rotational and inertial gravity modes based on the $3 \mathrm{D}$ normal mode with operational analyses data of ECMWF. However, no study based on the 3D normal mode decomposition has been carried out about the energy spectrum of gravity mode with high resolution analysis data.

Recently, the resolution of the analysis data is increasing very rapidly, because of the development of the atmospheric general circulation model, the data assimilation system, and also the computer technology. The purpose of this study is to investigate the characteristics of the energy spectrum of gravity mode with the high resolution analysis data provided by JMA, and to examine whether the transition of energy slope can be seen. In Section 2, the analysis method and description about dataset used in this study are described. In Section 3, the result of the energy spectrum in the zonal wavenumber domain is presented. Finally, the concluding remarks are given in Section 4.

\section{Analysis method and data}

In this section, we investigate the characteristics of spectral slope based on 3D normal mode energetics. The 3D normal mode energetics was constructed by Tanaka (1985). The basis function used in this study is 3D normal mode functions, which is defined by the tensor product of vertical structure functions and Hough functions. Expanding the momentum equations and thermodynamic equation, we can obtain the prognostic equation in $3 \mathrm{D}$ spectral domain as follows; 


$$
\frac{d w_{i}}{d \tau}=-i \sigma_{i} w_{i}-i \sum_{j, k}^{N} r_{i j k} w_{j} w_{k}+f_{i},
$$

where $w_{i}$ is a non-dimensional 3D normal mode expansion coefficient, $\tau$ is a non-dimensional time scaled by the angular speed of the Earth, $r_{i j k}$ is a nonlinear interaction coefficient, $\sigma_{i}$ is an eigenfrequency of Laplace's tidal equation, $i$ is an imaginary unit, and $f_{i}$ is a physical term, which includes diabatic heating and viscosity. For simplicity, $w_{n l m}$ is replaced by $w_{i}$, the subscripts $j$ and $k$ mean the different combinations of wavenumbers in 3D spectral space.

The subscripts $k, l$, and $m$ show zonal wavenumber, meridional mode number, and vertical mode number, respectively. The vertical mode $m=0$ is called a barotropic mode because the values of the mode are approximately constant and have no node in the vertical. The vertical mode $m=1$ has one node in the vertical, $m=$ 2 has two nodes and so on.

In this study, the truncation wavenumber in zonal, meridional, and vertical directions are set to 750,900 , and 30, respectively. The 900 Hough modes are divided into 3 parts; Rossby modes, eastward gravity modes, and westward gravity modes.

We can calculate total energy $E_{k l m}$ of each mode by using the spectral coefficient $w_{i}$,

$$
\begin{aligned}
& E_{k l m}=\frac{1}{2} p_{s} h_{m}\left|w_{k l m}\right|^{2}, \\
& E_{0 l m}=\frac{1}{4} p_{s} h_{m}\left|w_{0 l m}\right|^{2},
\end{aligned}
$$

where $p_{s}$ is the mean surface pressure $(1013.25 \mathrm{hPa})$ and $h_{m}$ is an equivalent height, which is obtained as an eigenvalue of the vertical structure equation. The units of energy are $\mathrm{J} \mathrm{m}^{-2}$.

The data used in this study are global $\eta$ surface analysis data provided by JMA (Japan Meteorological Agency) (JMA 2007). They are provided by way of "Meteorological Research Consortium", a framework for research cooperation between JMA and MSJ (Meteorological Society of Japan). The resolution of the model is TL959L60 (the model top is $0.1 \mathrm{hPa}$ ), corresponding roughly to a $20 \mathrm{~km}$ horizontal grid spacing. The data contain meteorological variables of horizontal wind $u, v$, geopotential $\phi$, specific humidity $q$, and air temperature $T$. The data period used in this study is from 00Z 1 September 2009 to $18 \mathrm{Z} 7$ September 2009. The data are interpolated on 60 gaussian vertical levels in the $-\log \left(p / p_{s}\right)$ coordinate by cubic spline method, the top of the gaussian vertical level is $0.4 \mathrm{hPa}$.

\section{Results}

Figure 1 shows the energy spectrum in the zonal wavenumber domain. The energy spectra of Rossby and gravity modes are obtained by summing up all vertical and meridional modes in Hough modes, respectively. The energy spectrum of Rossby modes follows -3 power law in the synoptic scale $(k=6$ to 300$)$. The slope of a linear regression for $k=6$ to 300 is -2.84 . It is found that the energy spectrum of gravity mode exactly obeys the $-5 / 3$ power law in both synoptic and mesoscales. The slope of a linear regression for $k=6$ to 350 is -1.66 . The spectra for Rossby and gravity modes become less steeper than $k^{-3}$ and $k^{-5 / 3}$ slopes from around $k=300$. The spectrum behind the wavenumber $k=$ 600 drops rapidly, because of the truncation and the viscosity and diffusion.

Nastrom and Gage (1985) showed that the energy slope shifts from -3 power spectrum to $-5 / 3$ power spectrum in the boundary of synoptic and mesoscale with aircraft observational data. In this study, the shift of total energy spectrum can not be clearly seen in the spectrum of total (both Rossby and gravity) energy.

It can also be seen in Fig. 1 that the spectra for Rossby and gravity energy cross each other around a zonal wavenumber 80 , although the total energy spectrum does not clearly show this shift. This scale corresponds to about $350 \mathrm{~km}$ on $45^{\circ}$ circle. Figure

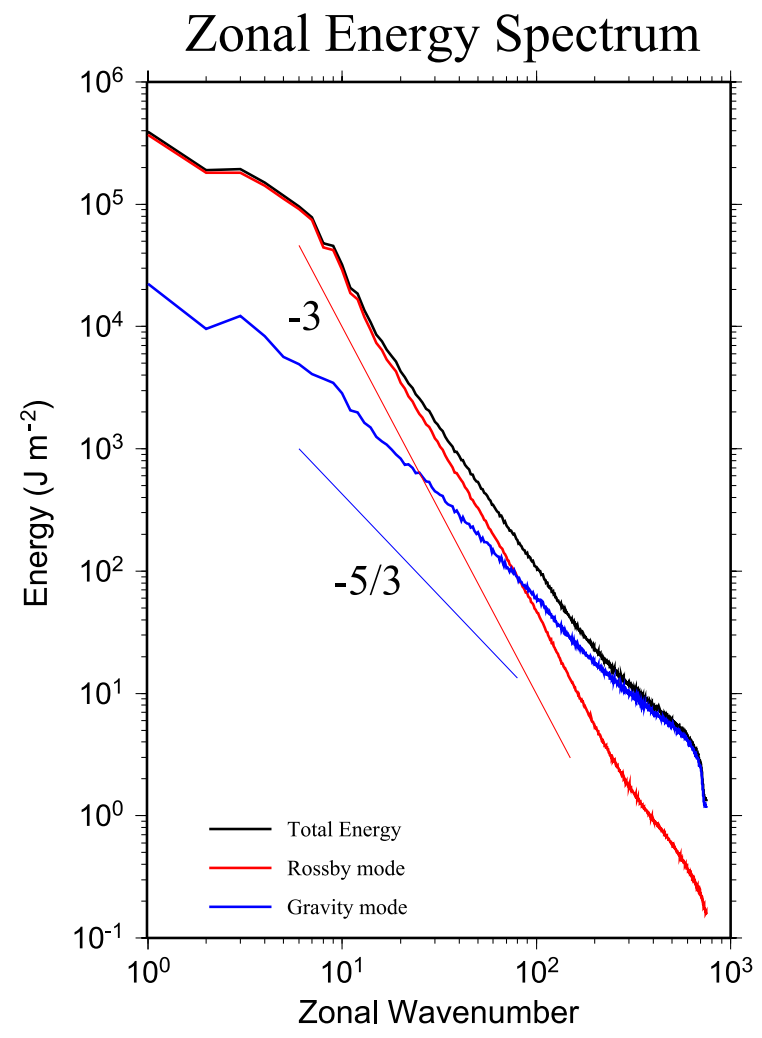

Fig. 1. The energy spectrum in zonal wavenumber domain. The black, red, and blue solid lines show total energy, energy of Rossby mode, and energy of gravity mode, respectively. The -3 and $5 / 3$ spectra are drawn by thin solid lines. The zonal wavenumber and energy are taken in the horizontal and vertical axes, respectively. The units of energy are $\mathrm{J} \mathrm{m}^{-2}$, and the zonal wavenumber is non-dimensional.

2 illustrates the ratio of Rossby wave energy to gravity wave energy. The Rossby (gravity) wave becomes dominant where the ratio is larger (smaller) than 1. This figure shows that the energy levels of Rossby mode and gravity mode become comparable where the energy slope shifts from $k^{-3}$ to $k^{-5 / 3}$. The characteristics of energy slopes agree very well with Nastrom and Gage's spectra which is based on the aircraft observational data.

Tanaka et al. (2004) proved that the energy spectrum in the phase speed domain is proportional to $c^{2}$, where $c$ is a nondimensional phase speed of Rossby wave $(c=\sigma / k)$. They showed a clear energy spectrum with reanalysis data, but up to $k=20$. Figure 3 illustrates the energy spectrum for barotropic mode up to $k=250$. The energy spectra of the same zonal wavenumber but the different meridional mode are connected with dotted line. The peak of the energy spectrum is seen around $c=10^{-2}\left(8.75 \mathrm{~m} \mathrm{~s}^{-1}\right.$ in $45^{\circ}$ circle). It seems that the spectral law based on the saturation of Rossby wave can be applied in small-scale phenomena. The spectrum in the phase speed domain follows $c^{2}$ law in $c \geq 10^{-5}$. The spectral slope in the small phase speed region becomes less steeper than $c^{2}$ and the edge spectrum has been eliminated in this study. It is desired to investigate in the future study.

\section{Conclusion and discussion}

In this study, the characteristics of the energy spectra for Rossby waves and gravity waves are investigated with the very high resolution analysis data (TL959L60) provided by JMA. In order to investigate the energy slope for Rossby waves and gravity waves, the 3D normal mode decomposition, which is derived by Tanaka (1985), is used. The focus of this study is on the transition 
Ratio of Rossby to Gravity modes

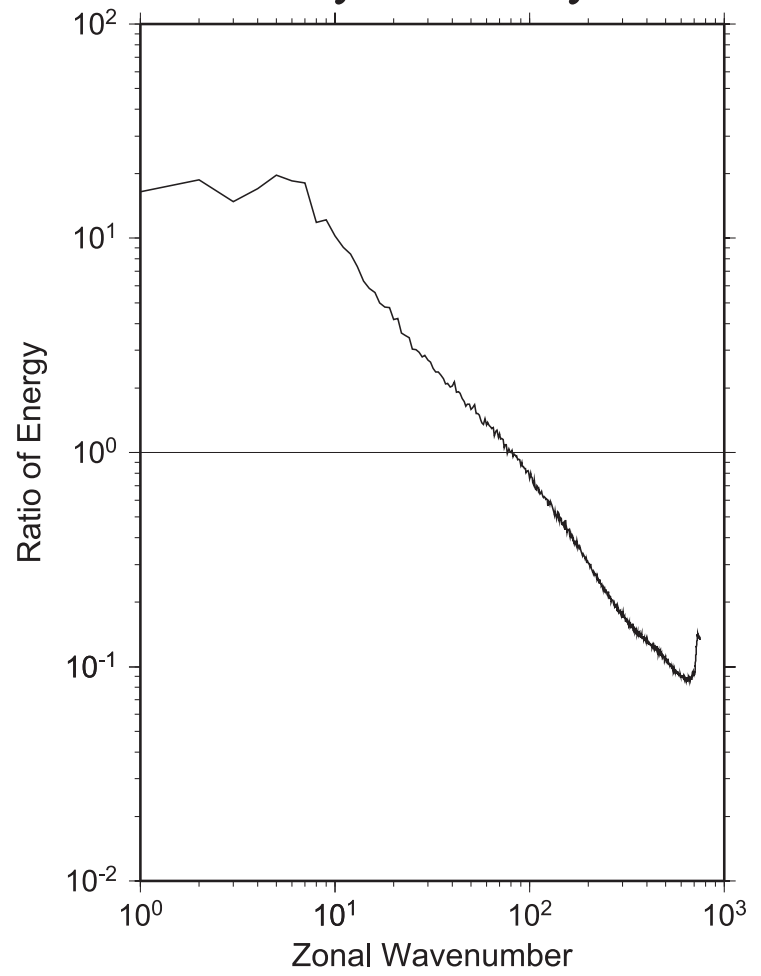

Fig. 2. The ratio of energy of the Rossby mode to gravity mode in Fig. 1.

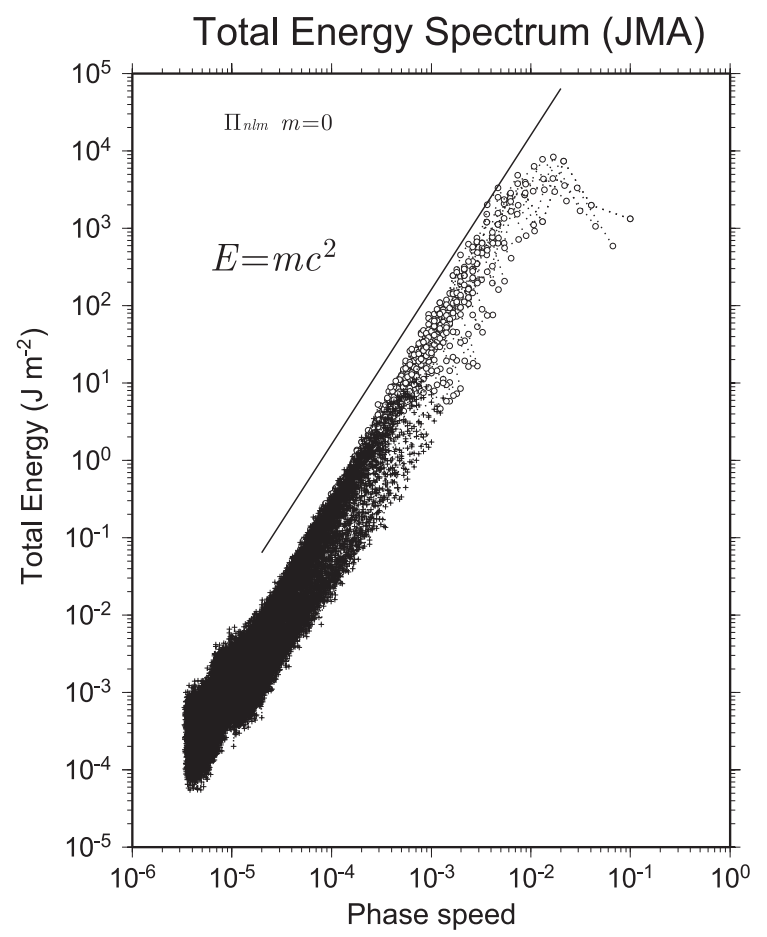

Fig. 3. The energy spectrum for the barotropic mode as a function of dimensionless phase speed of the Rossby mode $c$. Energy levels are connected by dotted lines for the same zonal wavenumber $k$ with different meridional mode numbers 1 . The spectrum of $E$ $=m c^{2}$ is drawn by solid line, where $m$ means a total mass of the atmosphere for unit area. The units of energy are $\mathrm{J} \mathrm{m}^{-2}$.

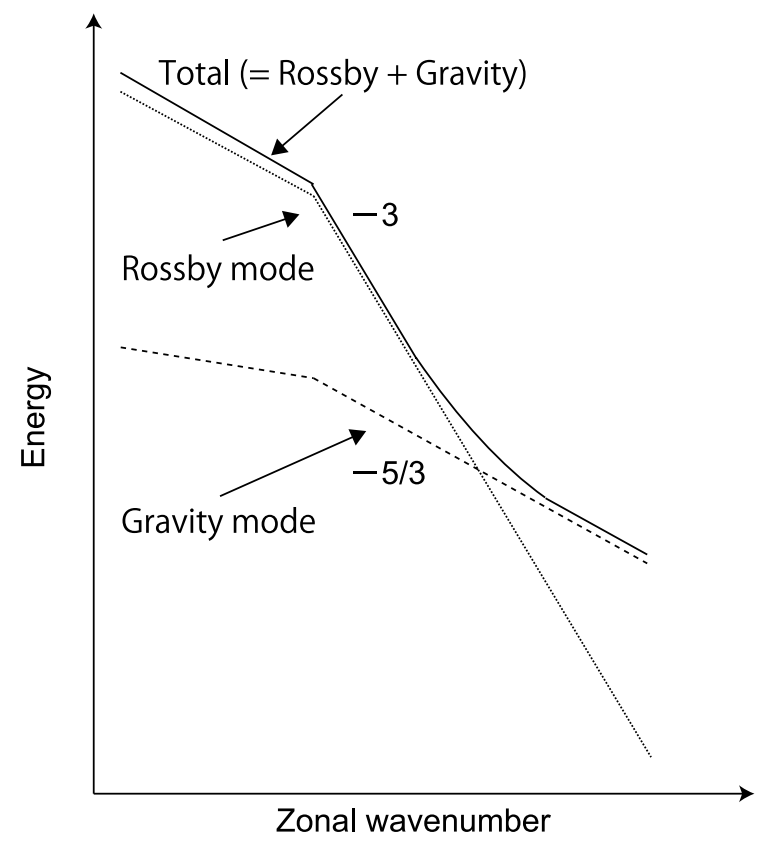

Fig. 4. Schematic diagram of energy spectrum for baroclinic atmosphere. The dotted and dashed lines show the energy spectra for Rossby and gravity modes, respectively. The solid line shows the total (Rossby + gravity) energy spectrum.

of energy slope from -3 to $-5 / 3$ regime in the zonal wavenumber domain.

Results show that the total energy spectrum does not clearly represent the transition of the energy slope, but the total energy spectrum shifts from $k^{-3}$ to $k^{-5 / 3}$ gradually. The energy level of Rossby waves is higher in the synoptic scale, and that of gravity waves becomes higher in the mesoscale. It is found in this study that the energy spectra for Rossby and gravity modes cross each other around $k=80$. It is essential for understanding these power laws in the general circulation that the energy spectra of Rossby and gravity waves obey the $k^{-3}$ and $k^{-5 / 3}$ laws, respectively. The schematic diagram of energy spectrum is shown in Fig. 4.

The ratio of Rossby wave energy to gravity wave energy clearly explains why the total energy shows the gradual spectral shift. The result of this study may indicate that the shifting wavenumber from $k^{-3}$ to $k^{-5 / 3}$ is determined just as a crossing wavenumber of Rossby wave and gravity wave spectra.

Terasaki et al. (2009) found that the energy spectrum of the vertical motion becomes white noise using NICAM experiment (3.5 km horizontal grid spacing), which has almost the same energy level at any horizontal scales. It is suggested that the $k^{-5 / 3}$ power spectral law in stratified turbulence may be explained by the saturation theory of the vertical motion. It is desired to examine the formation mechanism of energy spectrum in 3D normal mode energetics, and also to investigate the spectra with the high resolution model.

\section{Acknowledgement}

The data used in this study was provided by way of "Meteorological Research Consortium", a framework for research cooperation of JMA and MSJ. The computation of the 3D normal mode decomposition was carried out with the computer system installed in CCS (Center for Computational Sciences) in University of Tsukuba. 


\section{References}

Boer, G. J., and T. G. Shepherd, 1983: Large-scale two-dimensional turbulence in the atmosphere. J. Atmos. Sci., 40, 164184.

Hamilton, K., Y. O. Takahashi, and W. Ohfuchi, 2008: Mesoscale spectrum of atmospheric motions investigated in a very fine resolution global general circulation model. J. Geophys. Res., 113, D18110, doi;10.1029/2008JD009785.

JMA, 2007: Outline of the operational numerical weather prediction at Japan Meteorological Agency, $194 \mathrm{pp}$.

Kitamura, Y., and Y. Matsuda, 2006: The $k_{H}^{-3}$ and $k_{H}^{-5 / 3}$ energy spectra instratified turbulence. Geophys. Res. Lett., 33, L05809, doi:10.1029/2005GL024996.

Kitamura, Y., and Y. Matsuda, 2010: Energy cascade processes in rotating stratified turbulence with application to the atmospheric mesoscale. Geophys. Res. Lett., 115, L11104, doi: 10.1029/2009JD012368.

Kolmogorov, A., 1941: Dissipation of energy in the locally isotropic turbulence (English translation 1991). Proc. Roy. Soc. London, A434, 15-17.

Koshyk, J. N., and K. Hamilton, 2001: The horizontal kinetic energy spectrum and spectral budget simulated by a highresolution troposphere-stratosphere-mesosphere GCM. J. Atmos. Sci., 58, 329-348.

Kraichnan, R. H., 1967: Inertial ranges in two-dimensional turbulence. Phys. Fluids, 10, 1417-1423.

Nastrom, G. D., and K. S. Gage, 1985: A climatology of atmospheric wavenumber spectra of wind and temperature observed by commercial aircraft. J. Atmos. Sci., 42, 950960.

Nastrom, G. D., K. S. Gage, and W. H. Jaspersom, 1984: The atmospheric kinetic energy spectrum, $10^{0}-10^{4} \mathrm{~km}$. Nature, 310, 36-38.

Takahashi, Y. O., K. Hamilton, and W. Ohfuchi, 2006: Explicit global simulation of the mesoscale spectrum of atmospheric motions. Geophys. Res. Lett., 33, L12812, doi:10.1029/2006 GL026429.

Tanaka, H. L., 1985: Global energetics analysis by expansion into three dimensional normal mode functions during the FGGE winter. J. Meteor. Soc. Japan, 63, 180-200.

Tanaka, H. L., and E. C. Kung, 1988: Normal mode energetics of the general circulation during the FGGE year. J. Atmos. Sci., 45, 3723-3736.

Tanaka, H. L., Y. Watarai, and T. Kanda, 2004: Energy spectrum proportional to the squared phase speed of Rossby modes in the general circulation of the atmosphere. Geophys. Res. Lett., 31(13), 13109, doi:10.1029/2004GL019826.

Terasaki, K., and H. L. Tanaka, 2007a: Barotropic energy spectrum by the Rossby wave saturation in the zonal wavenumber domain. SOLA, 3, 25-28.

Terasaki, K., and H. L. Tanaka, 2007b: An analysis of the 3D atmospheric energy spectra and interactions using analytical vertical structure functions and two reanalyses. J. Meteor. Soc. Japan, 85, 785-796.

Terasaki, K., and H. L. Tanaka, and M. Satoh, 2009: Characteristics of the kinetic energy spectrum of NICAM model atmosphere. SOLA, 5, 180-183.

Tung, K. K., and W. W. Orland, 2003: The $k^{-3}$ and $k^{-5 / 3}$ energy spectrum of atmospheric turbulence: Quasigeostrophic twolevel model simulation. J. Atmos. Sci., 60, 824-835.

Wiin-Nielsen, A., 1967: On the annual variation and spectral distribution of atmospheric energy. Tellus, 23, 207-217.

Žagar, N., J. Tribbia, J. L. Anderson, and K. Rader, 2009: Uncertainties of estimates of inertia-gravity energy in the tmosphere. Part I: Intercomparison of four analysis systems. Mon. Wea. Rev., 137, 3878-3857.

Žagar, N., J. Tribbia, J. L. Anderson, and K. Rader, 2010: CORRIGENDUM. Mon. Wea. Rev., 138, 2476-2477.

Manuscript received 13 December 2010, accepted 22 February 2011 SOLA: http://www.jstage.jst.go.jp/browse/sola 